diet (MeDi) includes many components that might be beneficial for cognitive performance, such as high intakes of fruit, vegetables, unsaturated fatty acids and fish, moderate amounts of alcohol, and low intakes of saturated fatty acids, meat and dairy products. In a recent study, researchers used data from the Washington Heights-Inwood Columbia Aging Project to investigate whether greater MeDi adherence reduces AD risk.

MeDi scores (ranging from 1 to 9, with higher numbers indicating greater MeDi adherence) were generated for each subject from baseline data $(n=2,258)$. The primary outcome was the incidence of $A D$ (assessed approximately every 1.5 years). Analysis of data from a subset of 479 subjects with multiple dietary assessments indicated that MeDi scores were stable over time.

Over a mean follow-up of about 4 years, 262 individuals developed AD. Higher adherence to the MeDi at baseline was associated with a significantly lower risk for AD in unadjusted and adjusted models (adjusted hazard ratio $0.91 ; P=0.015)$. Compared with patients in the lowest tertile of MeDi adherence, subjects with medium and high adherences showed 15-21\% and $39-40 \%$ lower risks for AD development, respectively. In models examining the contribution of individual MeDi components, no individual component was by itself a significant predictor of AD.

The authors conclude that higher adherence to the MeDi is associated with a reduced risk of $A D$ development. The overall MeDi pattern seems to have a greater effect on risk of $A D$ than its individual components.

Original article Scarmeas $\mathrm{N}$ et al. (2006) Mediterranean diet and risk for Alzheimer's disease. Ann Neurol 59: 912-921

\section{Combining prestroke treatments reduces stroke severity}

Antiplatelet agents, angiotensin-convertingenzyme inhibitors and statins are well known to be effective in stroke prevention. A retrospective study by researchers at the Beth Israel Deaconess Medical Center has shown that, if these drugs are used in combination prior to stroke, stroke severity might be reduced further and the volume of ischemic tissue at risk decreased.

All patients in the study presented within $24 \mathrm{~h}$ of stroke onset. The 20 patients on all three stroke prevention therapies at stroke onset had a significantly lower NIS Stroke Scale score
$(P=0.001)$ and smaller perfusion-diffusion mismatch lesion volumes-indicating smaller volumes of ischemic tissue at risk-than the 43 patients on an antiplatelet agent plus a statin or angiotensin-converting-enzyme inhibitor, the 47 patients on antiplatelets alone, and the 69 patients taking no stroke treatment. Patients taking triple therapy were also more likely to have a shorter length of hospital stay and better functional status upon discharge, although this was probably due to differences in the initial severity of stroke rather than differences in the effect of the treatments on recovery.

The authors state that the additive effects of the combined therapies are probably caused by their differing modes of action. The findings of this study are preliminary and cannot lead directly to a recommendation for triple therapy in all patients at risk for stroke, but they nevertheless provide an important basis for further investigation.

Original article Kumar S et al. (2006) Antiplatelets, ACE inhibitors, and statins combination reduces stroke severity and tissue at risk. Neurology 66: 1153-1158

\section{Warfarin is not appropriate in all patients with AF-associated ischemic stroke}

Warfarin considerably reduces the risk of recurrent stroke in patients who have had an ischemic stroke and are in atrial fibrillation (AF). Researchers in New Zealand have investigated the reasons why, despite the proven benefits, only about $50 \%$ of patients with AF are started on warfarin therapy following ischemic stroke.

The analysis included 93 patients (mean age 80 years) admitted to Auckland City Hospital with ischemic stroke and either permanent or paroxysmal AF. The diagnosis of AF was known in 64 (69\%) patients before admission; the other patients were diagnosed during admission. Of the 64 patients in the former group, just 13 (20\%) were taking warfarin. In total, 17 patients died in hospital. At discharge, warfarin was recommended in 35 of the 76 surviving patients: 28 were already taking warfarin, 4 started warfarin after discharge and 3 did not commence treatment. Compared with those taking warfarin, patients who did not take warfarin were more likely to be dependent on others or to require institutional care at discharge. The most common reasons for not starting warfarin at 